TITLE:

\title{
Analytical end-to-end PER performance of multi-hop cooperative relaying and its experimental verification
}

\section{AUTHOR(S):}

MURATA, Hidekazu; MIYAGOSHI, Makoto; OISHI, Yuji

\section{CITATION:}

MURATA, Hidekazu ...[et al]. Analytical end-to-end PER performance of multi-hop cooperative relaying and its experimental verification. IEICE Transactions on Communications 2017, E100.B(3): 449-455

ISSUE DATE:

2017-03-01

URL:

http://hdl.handle.net/2433/260587

\section{RIGHT:}

(C) 2017 The Institute of Electronics, Information and Communication Engineers; 許諾条件に基づいて掲載しています。 


\title{
PAPER \\ Analytical End-to-End PER Performance of Multi-Hop Cooperative Relaying and Its Experimental Verification
}

\author{
Hidekazu MURATA $^{\dagger a}$, Senior Member, Makoto MIYAGOSHI ${ }^{\dagger *}$, Nonmember, and Yuji OISHI $^{\dagger * *}$, Member $^{\circ}$
}

SUMMARY The end-to-end packet error rate (PER) performance of a multi-hop cooperative relaying system is discussed in this paper. In this system, the end-to-end PER performance improves with the number of hops under certain conditions. The PER performance of multi-hop cooperative networks is analyzed with the state transition technique. The theoretical analysis reveals that the PER performance can be kept almost constant, or even improved, as the number of hops is increased. Computer simulation results agree closely with the analysis results. Moreover, to confirm this performance characteristic in an actual setup, an in-lab experiment using a fading emulator was conducted. The experimental results confirm the theoretical end-to-end PER performance of this system.

key words: cooperative relaying, space-time coding, multi-hop relaying, transmission experiment

\section{Introduction}

One promising technology for enhancing the performance of wireless networks is the multi-hop wireless network [1]-[6], where relay stations forward data from a source station to a destination station. The major advantages of the multi-hop wireless network are the extension of coverage and mitigation of the shadowing effect. However, the end-to-end packet error rate (PER) performance of this system with a simple relaying scheme is degraded with the increasing number of hops due to the error propagation through the hops.

To cope with such degradation and improve the performance of the multi-hop wireless network, cooperation among relay stations is proposed [7]. Cooperative relay stations work together using transmit diversity, and thus reduce errors through the diversity effect. In these networks, an error at a relay station can be recovered at the next hop unless all the relay stations at each hop do not receive a packet correctly, and the end-to-end error performance is improved compared to normal (one relay station at each hop) relaying networks.

In this system, the end-to-end PER performance can improve with the number of hops while keeping the signal to noise ratio (SNR) at each hop constant. To address this unique characteristic, the end-to-end packet error rate (PER) performance of this multi-hop cooperative relaying system is discussed in this paper. The contribution of this paper is

Manuscript received March 30, 2016.

Manuscript revised September 7, 2016.

Manuscript publicized October 12, 2016.

$\dagger$ The authors are with the Graduate School of Informatics, Kyoto University, Kyoto-shi, 606-8501 Japan.

${ }^{*}$ Presently, with NTT DOCOMO INC.

** Presently, with Hitachi, Ltd., Research \& Development Group.

a) E-mail: contact-h28j@hanase.kuee.kyoto-u.ac.jp

DOI: $10.1587 /$ transcom.2016EBP3132 threefold: First, the theoretical analysis reveals that the PER performance can be kept almost constant, or even improved with an increasing number of hops. Second, the computer simulation results agree closely with the analysis results. Finally, the experimental results confirm the theoretical endto-end PER performance of this system.

First, we derive the theoretical end-to-end PER performance of multi-hop cooperative networks using transmit diversity and evaluate the performance with numerical examples [8], [9]. This system consists of a source, a destination, and more than one cooperative relay in each hop. Relays are assumed to decode their received packets and forward them only when no error is found. The end-to-end PER performance of the cooperative relaying system improves with the number of hops under some conditions.

In real-world wireless systems, however, various practical issues, such as imperfect channel estimation and hardware imperfections, have a considerable impact on the performance, especially on the efficiency of transmit diversity. The goal of this study is to evaluate the end-to-end PER performance of the multi-hop cooperative relaying system with actual transmission equipment. Many papers have discussed the implementation and experimental results of cooperative communications [10]-[13]. However, to the best of the authors' knowledge, evaluations of experimental errors for the cooperative multi-hop (more than 2-hop) transmission have not been reported. Experimental results of cooperative multi-hop transmission were reported in [14], but that study focused on the timing synchronization technique.

This paper performs in-lab experiments using a fading emulator and four transceivers to measure the end-to-end PER performance of the multi-hop cooperative relaying system under a Rayleigh fading environment [15] to confirm the theoretical results.

\section{Theoretical Analysis of Multi-Hop Cooperative Net- works}

\subsection{System Model}

Figure 1 shows the system model considered in this paper. The multi-hop cooperative network consists of the source station, $K$ cooperative relay stations at each hop and the destination station. Each relay station forwards a received packet if it is correctly decoded. All channels connecting the transmitting stations and the receiving stations are assumed to be independent and identically distributed (i.i.d.) 


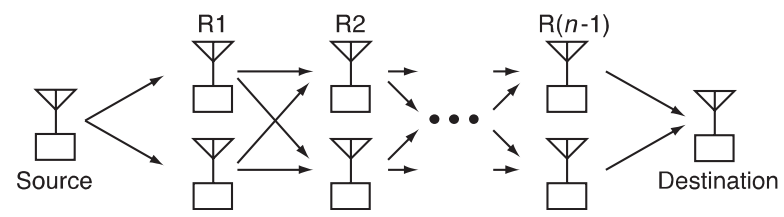

Fig. 1 Cooperative $n$-hop relaying system $(K=2)$.

Rayleigh fading channels. The transmission power of the cooperative relay stations and the distance of each hop are assumed to be equal across the entire network. Error detection at the receivers is assumed to be ideal. In this paper, the performance is measured in terms of the end-to-end PER.

It is assumed that packet transmission at each hop is free from interference from other hops. Basically, for an $N$-hop network it is necessary to provide $N$ unique radio resource units to avoid interference among hops. In this network model, throughput degradation is a widely known issue and depends on a spatial reuse scheme of the radio resource. Therefore, this throughput degradation should be studied with routing algorithms [16], [17]. Furthermore, the transmission performance of multi-hop cooperative networks in the presence of interference should be carefully studied [18]. However, these are outside of the scope of this paper.

\subsection{Two Relay Stations in Each Hop}

For the system with two $(K=2)$ cooperative relay stations in each hop, three cases are considered:

1. Two stations forward the signal.

2. Only one station forwards the signal.

3. Neither station forwards the signal.

The behavior of the multi-hop cooperative communication system can be modeled as a state transition among these three states. The state diagram is shown in Fig. 2. State $s_{i}(i=$ $0,1,2)$ denotes that there are $i$ transmitting relay stations. The expression at each branch denotes the conditional probability that the state transits between states, where $F_{2}$ and $F_{1}$ are the average PERs when two stations transmit and one station transmits, respectively. Obviously, $0<F_{2}<F_{1}<1$ due to the transmit diversity gain. For example, the transition from $s_{2}$ to $s_{2}$ represents the case where two stations transmit and two stations receive without error. Because the average PER is $F_{2}$, the conditional probability that a station receives a packet correctly is $\left(1-F_{2}\right)$. If two stations receive the packet, the conditional probability becomes $\left(1-F_{2}\right)^{2}$, because their error probabilities are assumed to be independent. $n$th hop

Let us consider the state probability distribution at the

$$
\boldsymbol{p}_{n}=\left[p_{2}, p_{1}, p_{0}\right]^{\mathrm{T}},
$$

where $p_{i}(i=0,1,2)$ denotes the probability of state $s_{i}$. From Fig. 2, by using the state probability distribution of the $n$th hop, the state probability distribution of the $(n+1)$ th hop

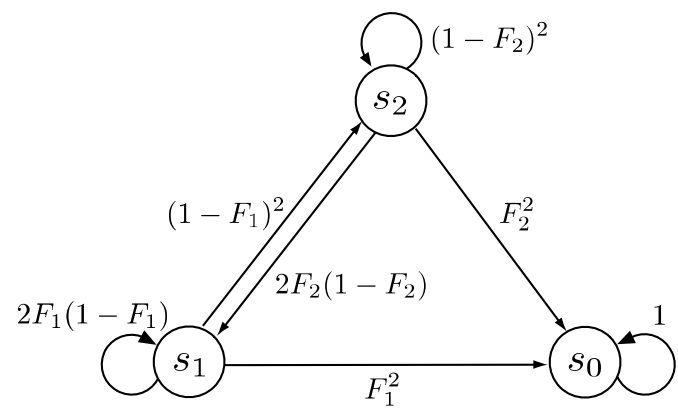

Fig. 2 State diagram. States $s_{0}, s_{1}, s_{2}$ denote the number of transmit stations as $0,1,2$, respectively, and the expression at each branch denotes the conditional probability of the state transition.

can be written as:

$$
\begin{aligned}
\boldsymbol{p}_{n+1} & =\left[\begin{array}{ccc}
\left(1-F_{2}\right)^{2} & \left(1-F_{1}\right)^{2} & 0 \\
2 F_{2}\left(1-F_{2}\right) & 2 F_{1}\left(1-F_{1}\right) & 0 \\
F_{2}^{2} & F_{1}^{2} & 1
\end{array}\right] \boldsymbol{p}_{n} \\
& =\boldsymbol{X} \boldsymbol{p}_{n} .
\end{aligned}
$$

By considering the errors that occurred at the last hop, the end-to-end PER performance after $N(\geq 2)$ hops is written as:

$$
\begin{aligned}
P E R & =\left[\begin{array}{lll}
F_{2} & F_{1} & 1
\end{array}\right] \boldsymbol{p}_{N} \\
& =\left[\begin{array}{lll}
F_{2} & F_{1} & 1
\end{array}\right] \boldsymbol{X}^{N-2} \boldsymbol{p}_{2} \\
& =\left[\begin{array}{lll}
F_{2} & F_{1} & 1
\end{array}\right] \boldsymbol{X}^{N-2}\left[\begin{array}{c}
\left(1-F_{1}\right)^{2} \\
2 F_{1}\left(1-F_{1}\right) \\
F_{1}^{2}
\end{array}\right],
\end{aligned}
$$

where $\left[\begin{array}{lll}F_{2} & F_{1} & 1\end{array}\right]$ and $\boldsymbol{p}_{2}$ denote the error probabilities at the last and the first hops, respectively.

Let us consider the state diagram of Fig. 2 again. States $s_{1}$ and $s_{2}$ can transit to all the states. Even if the state is $s_{1}$, which represents the case where only one station forwards the signal, the state can transit to $s_{2}$ when two stations at the next hop can receive packets correctly. Then the network recovers to a better state at further hops, and the end-to-end error may not occur. However, once the state transits to $s_{0}$, which represents the case where both relay stations detect errors and stop their transmission, the state never goes to $s_{2}$ or $s_{1}$, because neither station at the next hop can receive the packet. So, $p_{0}$ monotonically increases with the increasing number of hops and always results in an end-to-end error. Because states $s_{1}$ and $s_{2}$ make fewer errors than $s_{0}$, the major source of errors is $s_{0}$, especially when the hop count is large. When the hop count is small, the initial state probability distribution $\boldsymbol{p}_{2}$ dominates the error performance.

\section{3 $K$ Relay Stations in Each Hop}

Similarly, the PER performance of the networks that have $K$ relay stations can be derived. For $K+1$ states $s_{K}, \cdots, s_{0}$, the state probabilities are $p_{K}, \cdots, p_{0}$, respectively. The $(i, j)$ element of the $(K+1) \times(K+1)$ state transition matrix $\boldsymbol{X}$ 
Table 1 Computer simulation parameters

\begin{tabular}{lr}
\hline Parameter & Value \\
\hline Modulation scheme & QPSK \\
Packet length & 128 symbols \\
Channel model & i.i.d. Rayleigh fading \\
& Jakes' model \\
$f_{\mathrm{D}} T_{\mathrm{s}}$ & $1 / 5000$ \\
Channel estimation & Perfect \\
Demodulation & MLSE \\
\hline
\end{tabular}

becomes:

$$
X_{i j}={ }_{K} C_{K-i+1} F_{K-j+1}^{i-1}\left(1-F_{K-j+1}\right)^{K-i+1},
$$

where $1 \leq i, j \leq K+1$ and $F_{0}=1 . X_{i j}$ gives the conditional probability that $K-i+1$ stations receive correctly when $K-j+1$ stations transmit. This is the binomial expansion of $F_{K-j+1}$ and $1-F_{K-j+1}$, and the summation about $i$ becomes 1. The error probabilities of the first and the last hops are written as:

$$
\boldsymbol{p}_{2}=\left[\begin{array}{c}
\left(1-F_{1}\right)^{K} \\
K F_{1}\left(1-F_{1}\right)^{K-1} \\
\vdots \\
{ }_{K} C_{K-i+1} F_{1}^{i-1}\left(1-F_{1}\right)^{K-i+1} \\
\vdots \\
F_{1}^{K}
\end{array}\right]
$$

and

$$
p_{\text {last }}=\left[\begin{array}{llllll}
F_{K} & F_{K-1} & \cdots & F_{K-i+1} & \cdots & 1
\end{array}\right]
$$

respectively. Thus, $P E R$ is written as

$$
P E R=\boldsymbol{p}_{\text {last }} \boldsymbol{X}^{N-2} \boldsymbol{p}_{2} .
$$

\section{Numerical Examples}

In the following, QPSK delay diversity transmission is assumed. Figures 3 and 5 show the state probability versus hop count. Figures 4 and 6 show the theoretical PER performance, contribution of each state to the performance, and the computer simulation results. Each curve, except the one indicated "Total", is the PER due to each state, given as $F_{i} p_{i}$ $\left(i=0,1,2, F_{0}=1\right)$. The total PER is defined as the sum of the PER of each state. The simulation parameters are listed in Table 1 .

The first example assumes two relay stations and average $E_{\mathrm{b}} / N_{0}=17 \mathrm{~dB}$ when one station transmits, that is, $F_{1} \approx 0.07$ and $F_{2} \approx 0.003$. In Fig. $3, p_{2}$ is almost 1 and is much larger than $p_{1}$ and $p_{0}$. Also, $p_{1}$ decreases significantly with increasing number of hops when the hop count is small. However, $p_{0}$ does not increase much while $p_{2}$ increases. The decrease of $p_{1}$ and increase of $p_{2}$ reduce the total errors, and thus the PER performance is improved, as shown in Fig. 4. The PER has its minimum at a hop count of 5 and slowly increases with increasing number of hops. Figure 4 also shows that $s_{0}$ contributes the most to the PER, followed by

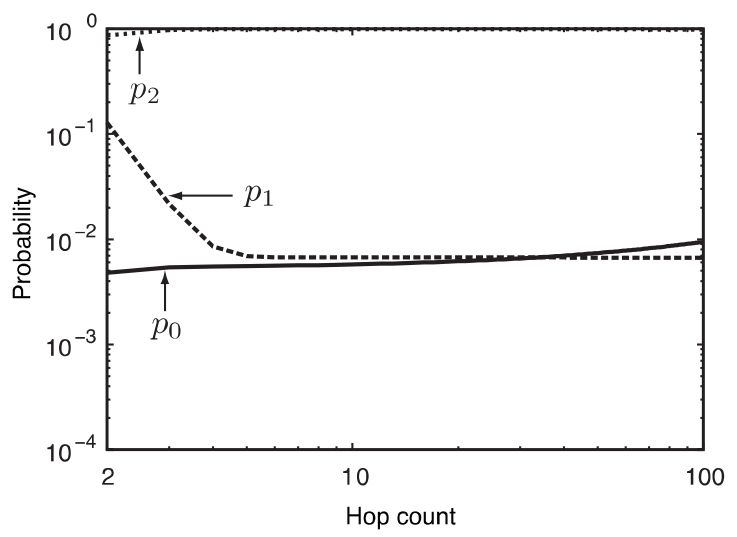

Fig. 3 State distribution, two relay stations, $E_{\mathrm{b}} / N_{0}=17 \mathrm{~dB}$.

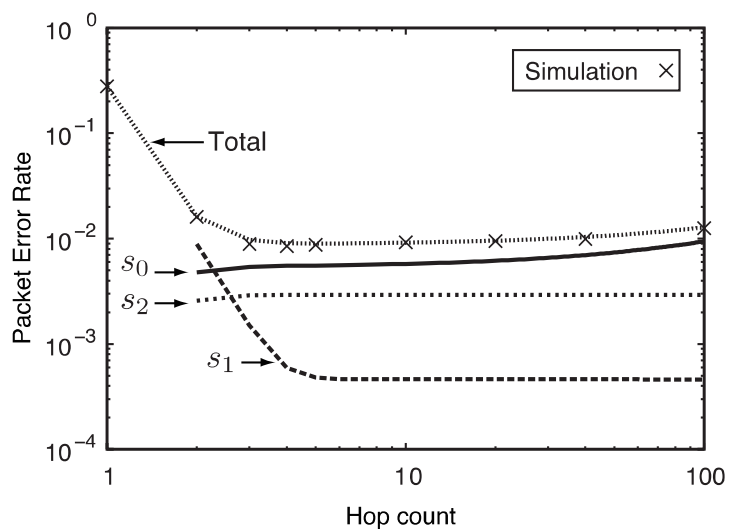

Fig. 4 Theoretical PER, contribution of each state, and simulation result, two relay stations, $E_{\mathrm{b}} / N_{0}=17 \mathrm{~dB}$.

$s_{2}$, because $s_{0}$ always makes an error, as mentioned in the previous section. Because $F_{2}$ is small, $s_{2}$, whose probability $p_{2}$ is more than 100 times larger than $p_{0}$, causes fewer errors than does $s_{0}$. Although $F_{1}$ is larger than $F_{2}, s_{1}$ causes much fewer errors than $s_{2}$, because $p_{1}$ is much smaller than $p_{2}$.

The second example assumes three relay stations and $E_{\mathrm{b}} / N_{0}=17 \mathrm{~dB}$ when one station transmits, and $F_{1} \approx 0.07$, $F_{2} \approx 0.003$ and $F_{3} \approx 5 \times 10^{-5}$. As shown in Fig. 5, $p_{1}$ and $p_{2}$ decrease significantly with the increasing number of hops, $p_{3}$ increases, and $p_{0}$ remains almost the same. It is also shown that the state distribution does not change when the packets are relayed many times. Most of the total PER is made by $s_{0}$ when the hop count is large, as shown in Fig. 6. When the hop count is small, $s_{1}$ contributes the most to the total PER, followed by $s_{2}$. Because $p_{1}$ and $p_{2}$ decrease significantly as packets are relayed, the total PER gets significantly lower when the hop count is small, and remains almost the same while packets are relayed many times.

Figure 7 shows the PER performance of the networks with two and three relay stations at each hop, when $E_{\mathrm{b}} / N_{0}=10 \mathrm{~dB}$. The PER performance of the network with two relay stations at each hop is degraded with the increasing number of hops. However, with three relay stations, the PER performance is improved with the increasing number of 


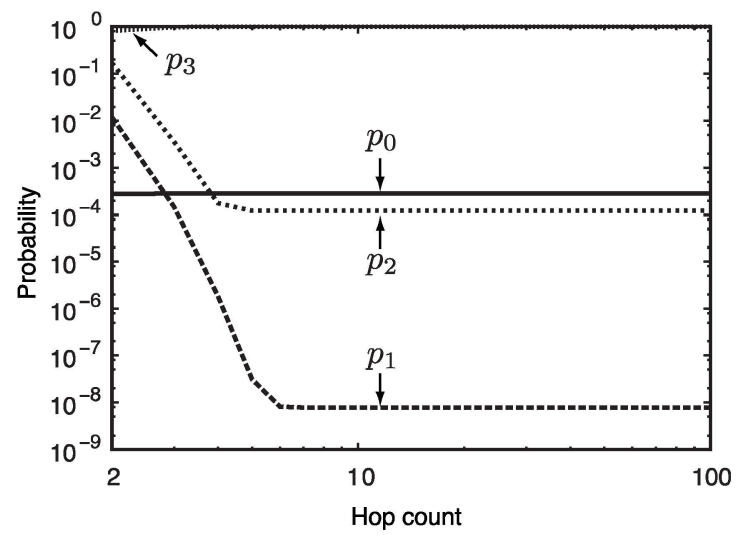

Fig. 5 State distribution, three relay stations, $E_{\mathrm{b}} / N_{0}=17 \mathrm{~dB}$.

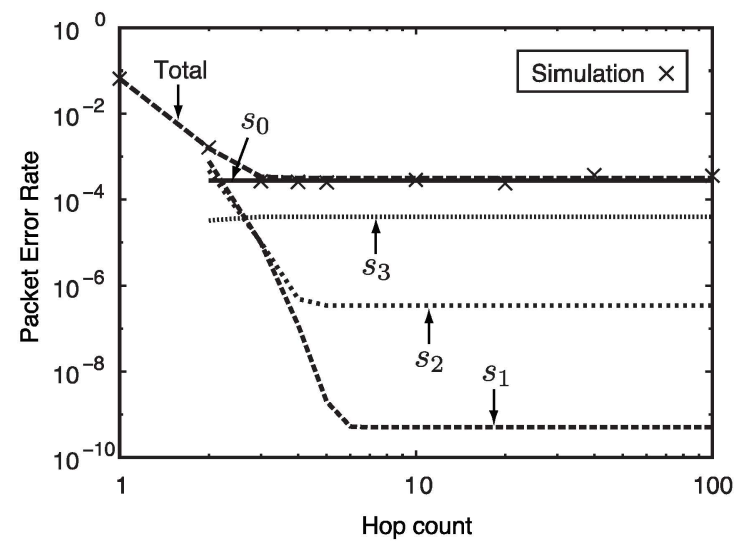

Fig. 6 Theoretical PER, contribution of each state, and simulation result, three relay stations, $E_{\mathrm{b}} / N_{0}=17 \mathrm{~dB}$.

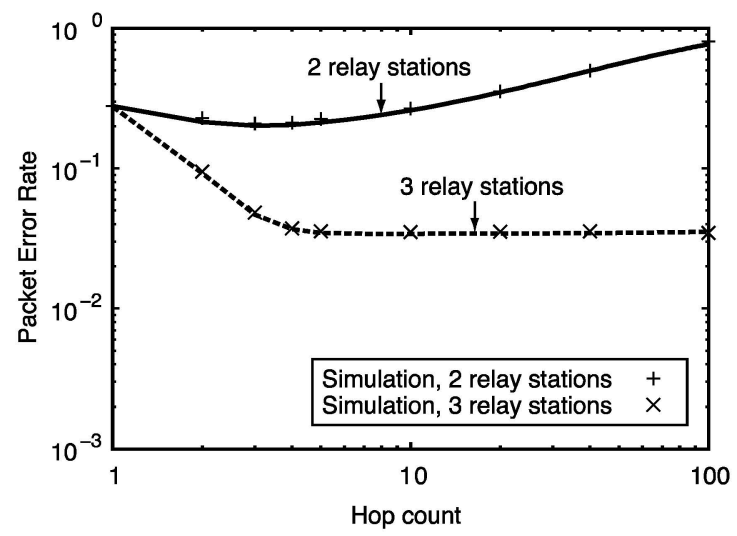

Fig. 7 Theoretical PER and simulation results, two and three relay stations, $E_{\mathrm{b}} / N_{0}=10 \mathrm{~dB}$.

hops when the hop count is small, and the PER performance remains almost the same when the hop count is large. The theoretical results closely agree with the simulation results in each example.

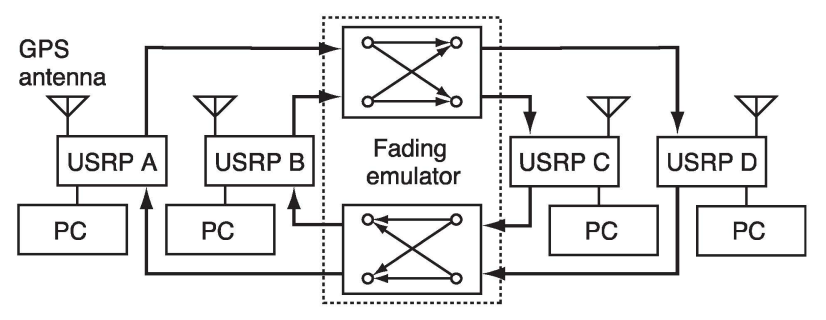

Fig. 8 Block diagram of experimental setup.

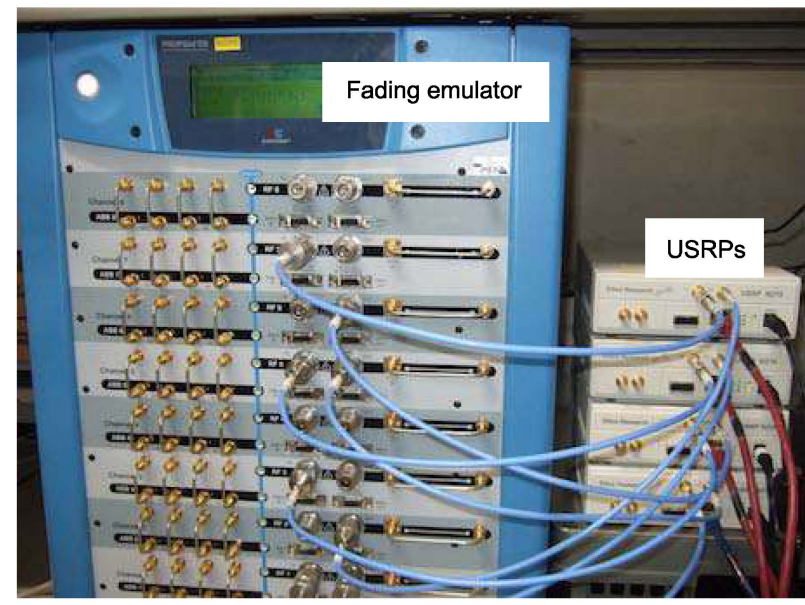

Fig.9 Experimental setup.

\section{Experimental Verification}

\subsection{Experimental Setup}

Figures 8 and 9 show the block diagram and a photo of the experimental setup, respectively. This experimental system consists of universal software radio peripheral (USRP) $\mathrm{N} 210$ s as transceivers, global positioning system (GPS) receivers for synchronizing the USRPs, PCs as processors for the digital baseband signals from the USRPs, and a fading emulator as propagation channels. Because our fading emulator can simultaneously emulate up to eight channels, this setup repeatedly utilizes two $2 \times 2$ channels, as shown in Fig. 8, and one loop is assumed to be two hops. In this system, USRP A first transmits as the source station. After finishing the cooperative relaying between USRP A, B and C, D nine times, both USRP A and B finally receive packets at 10 hops and do not forward them.

In the space-time block code (STBC) technique, timing and frequency synchronization is crucial. In this system, a one-pulse-per-second (1 PPS) signal and a frequency reference signal from a GPS disciplined oscillator (GPSDO) are utilized for timing and frequency synchronization.

\subsection{Signaling Format}

The signaling format is shown in Fig. 10. In our system, a general-purpose computer is used as the corresponding PC; 


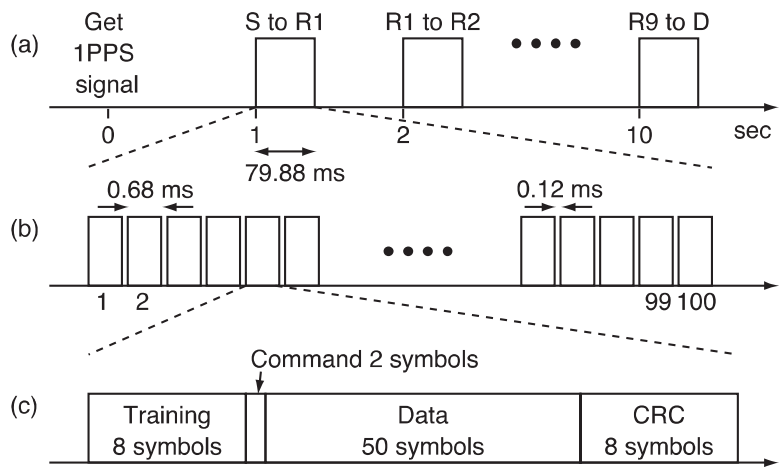

Fig. 10 Signaling format.
Table 2 Measurement system parameters.

\begin{tabular}{lr}
\hline Parameter & Value \\
\hline Number of hops & 10 \\
Radio frequency & $5.109375 \mathrm{GHz}$ \\
Modulation scheme & QPSK \\
Symbol rate & 100 symbols/s \\
Oversampling & 8 samples/symbol \\
Packet length & 68 symbols \\
Filter & Root roll-off Nyquist \\
& (Roll-off factor $\alpha=0.7$ ) \\
Receiver architecture & Low IF \\
STBC & Alamouti scheme \\
1 PPS acquisition & Every 11 seconds \\
Channel model & i.i.d. Rayleigh fading \\
& Classical Doppler spectrum \\
Maximum Doppler frequency & $5 \mathrm{~Hz}$ \\
\hline
\end{tabular}

\section{Experimental Results}

\subsection{Single-Hop Performance}

To confirm the performance of these implementations, the single-hop bit error rate (BER) and the PER are measured. In our system, the source station transmits using QPSK without STBC, and the relay stations transmit with STBC. In Sect. 2, the difference between PER performance without STBC and with STCB is ignored and both of them are simply shown as $F_{1}$ because no difference is expected with perfect CSI at the receiver. However, in our experimental system, the channel estimation at the receiver is not perfect. The performance $F_{1}$ with STBC can be degraded from that without STBC because the channel estimation is not perfect. To confirm this issue by transmission experiments, USRP A and B, shown in Fig. 8, transmit in three modes: both USRPs transmit with STBC, one USRP transmits with STBC, and one USRP transmits without STBC.

Figures 11 and 12 show the single-hop BER and PER measured at USRPC. The theoretical BER performance of QPSK without cooperation is also presented in Fig. 11. As shown in Fig. 11, the performance degradation due to channel estimation error, etc., is not severe for modes without cooperation. We can see from Figs. 11 and 12 that the difference between the non-cooperative transmission performance with STBC and without STBC is negligible. Also, we see from these figures that the error rate performance of STBC based cooperative transmission is better than that of noncooperative transmission.

\subsection{0-Hop End-to-End PER}

Figure 13 shows the result of the end-to-end PER measurement. In this experiment, PER is measured only when data transfer between the USRP and the control PC finishes on time through 10-hop transmissions. We use the same setting in the four USRPs. The average received SNR of the USRPs are different since these USRPs are not calibrated.

The end-to-end PER shown in Fig. 13 is the average values of two PERs measured on USRP A and B, or C and D in 
ets, the stations in the next hop can receive packets from another relay station. Also, we can see from Fig. 13 that the experimental end-to-end PER performance agrees with the theoretical end-to-end PER performance. Therefore, the theoretical end-to-end PER performance is verified by the experiments. The difference between the PER performance of even-numbered hops and that of odd-numbered hops in the experimental results may come from the imbalance in the performances among the USRPs.

\section{Conclusion}

This paper examined the end-to-end PER performance of the cooperative relay system. The theoretical PER performance of multi-hop cooperative networks is derived for any number of cooperative relays in each hop. The improvement in the end-to-end PER with the number of hops is demonstrated both theoretically and numerically. Experiments show that the end-to-end PER performance of this system can actually improve with the number of hops.

\section{Acknowledgment}

This research and development work was supported by the MIC/SCOPE \#165007006.

\section{References}

[1] A. Sendonaris, E. Erkip, and B. Aazhang, "Increasing uplink capacity via user cooperation diversity," IEEE International Symposium on Information Theory, p.156, Aug. 1998.

[2] Y.D. Lin and Y.C. Hsu, "Multihop cellular: A new architecture for wireless communications," IEEE INFOCOM 2000, pp.1273-1282, March 2000.

[3] J.N. Laneman and G.W. Wornell, "Energy-efficient antenna sharing and relaying for wireless networks," IEEE Wireless Communications and Networking Conference (WCNC), pp.7-12, Sept. 2000.

[4] Y. Yamao, T. Otsu, A. Fujiwara, H. Murata, and S. Yoshida, "Multihop radio access cellular concept for fourth-generation mobile communications system," IEEE International Symposium on Personal, Indoor and Mobile Radio Communications (PIMRC), pp.59-63, Sept. 2002.

[5] R. Pabst, B.H. Walk, D.C. Schultz, P. Herhold, H. Yanikomeroglu, S. Mukherjee, H. Viswanathan, M. Lott, W. Zirwas, M. Dohler, H. Aghvami, D.D. Falconer, and G.P. Fettweis, "Relay-based deployment concepts for wireless and mobile broadband cellular radio," IEEE Commun. Mag., vol.42, no.9, pp.80-89, Sept. 2004.

[6] J.N. Laneman, D.N.C. Tse, and G.W. Wornell, "Cooperative diversity in wireless networks: Efficient protocols and outage behavior," IEEE Trans. Inf. Theory, vol.50, no.12, pp.3062-3080, Dec. 2004.

[7] T. Miyano, H. Murata, and K. Araki, "Space time coded cooperative relaying technique for multihop communications," IEEE 60th Vehicular Technology Conference (VTC2004-Fall), pp.5140-5144, Sept. 2004.

[8] R. Fusawa, H. Murata, and K. Araki, "Theoretical FER performance of multihop wireless cooperative networks using transmit diversity," IEICE-CS Conf. IEICE'06, pp.B-5-121, Sept. 2006.

[9] Y. Oishi, H. Murata, K. Yamamoto, and S. Yoshida, "Theoretical FER performance of multi-hop wireless cooperative networks using transmit diversity," IEEE Vehicular Technology Conference, pp.2366-2369, May 2008.

[10] H. Murata, Y. Oishi, K. Yamamoto, and S. Yoshida, "FPGA implementation of STBC based cooperative relaying system," IEICE stem, each hop has two cooperative relay stations; therefore, even if one relay station does not forward pack- 
Trans. Commun., vol.E93-B, no.8, pp.1988-1992, Aug. 2010.

[11] P. Murphy and A. Sabharwal, "Design, implementation, and characterization of a cooperative communications system," IEEE Trans. Veh. Technol., vol.60, no.6, pp.2534-2544, July 2011.

[12] T. Korakis, M. Knox, E. Erkip, and S. Panwar, "Cooperative network implementation using open source platforms," IEEE Commun. Mag., no.2, pp.134-141, Feb. 2009.

[13] T. Mimura, A. Kuwabara, H. Murata, K. Yamamoto, and S. Yoshida, "Packet transmission experiments of STBC-based multi-hop cooperative relaying," IEEE International Conference on Communications, Kyoto, June 2011.

[14] Y.J. Chang and M.A. Ingram, "Convergence property of transmit time pre-synchronization for concurrent cooperative communication," IEEE Global Telecommunications Conference, Dec. 2010.

[15] M. Miyagoshi, H. Murata, S. Yoshida, K. Yamamoto, D. Umehara, S. Denno, and M. Morikura, "Experimental verification of PER performance of STBC-based multi-hop cooperative relaying," IEEE Vehicular Technology Conference (VTC2012-Fall), Sept. 2012.

[16] S. Chachulski, M. Jennings, S. Katti, and D. Katabi, "Trading structure for randomness in wireless opportunistic routing," ACM SIGCOMM'07, pp.169-180, Kyoto, Japan, Aug. 2007.

[17] S. Katti, D. Katabi, H. Balakrishnan, and M. Medard, "Symbol-level network coding for wireless mesh networks," ACM SIGCOMM'08, pp.401-412, Seattle, Washington, USA, Aug. 2008.

[18] T. Ohno, H. Murata, K. Yamamoto, and S. Yoshida, "Coexistence performance improvement of spectrum sharing system by using multihop cooperative relaying," Proc. the 7th International Conference on Information, Communications and Signal Processing (ICICS 2009), Macau, Dec. 2009.

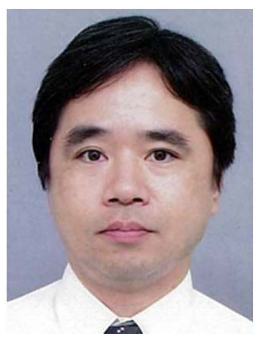

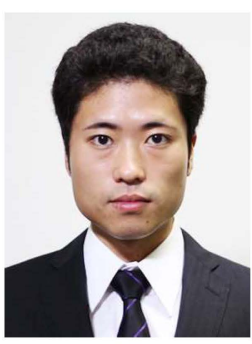

Yuji Oishi received his B.E. degree in Electrical and Electronic Engineering and his M.E. degree in Informatics from Kyoto University, Kyoto, Japan, in 2008 and 2010, respectively. In 2010, he joined Hitachi, Ltd., Research \& Development Group, Japan. major research interests include signal processing and its hardware implementation. He received the Young Researcher's Award from the IEICE of Japan in 1997, the Ericsson Young Scientist Award in 2000, and the Young Scientists' Prize for the Commendation for Science and Technology by the Minister of Education, Culture, Sports, Science and Technology in 2006, and the Paper Award of the IEICE in 2011 and 2013, and IEEE ICC Best Paper Award in 2014. He is a member of the ITE and IEEE.

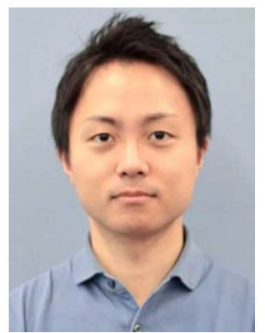

Makoto Miyagoshi received his B.E. degree in Electrical and Electronic Engineering and his M.E. degree in Informatics from Kyoto University, Kyoto, Japan, in 2011 and 2013, respectively. In 2013, he joined NTT DOCOMO, Yokosuka, Japan. 